\title{
Numerical study of the hadron-quark mixed phase
}

\author{
Tomoki Endo ${ }^{a *}$, Toshiki Maruyamab ${ }^{\mathrm{b}}$ Satoshi Chiba ${ }^{\mathrm{b}}$ and Toshitaka Tatsumi ${ }^{\mathrm{a}}$ \\ aDepartment of Physics, Kyoto University, Kyoto, 606-8502, Japan \\ b Advanced Science Research Center, Japan Atomic Energy Research Institute, Tokai, \\ Ibaraki, 319-1195, Japan
}

The Coulomb screening effect and the finite-size effect such as surface tension are figured out in the hadron-quark deconfinement phase transition. We study the mixed phase of the quark droplets immersed in hadron matter. We see that the droplet phase is mechanically unstable if the surface tension is strong enough. Once the Coulomb potential is properly taken into account, we could effectively satisfy the condition for charge chemical equilibrium in the Maxwell construction. As a result, we suggest the Maxwell construction revives the physical meaning effectively.

\section{Introduction}

Nowadays, it has been believed that hadron matter becomes quark matter in highdensity region, that is to say "deconfinement phase transition". We are here interested in how hadron matter changes to quark matter at zero temperature.

In the first-order phase transition, such as the water-vapor phase transition, we know the the Maxwell construction (MC) works to derive the equation of state in thermodynamic equilibrium. When we naively apply $\mathrm{MC}$ to this deconfinement phase transition, we immediately find one of the Gibbs conditions (GC) is not fulfilled, which are basic conditions in thermodynamics;

$\mu_{\mathrm{B}}^{\text {quark }}=\mu_{\mathrm{B}}^{\text {hadron }}\left(\equiv \mu_{\mathrm{B}}\right), \quad \mu_{\mathrm{e}}^{\text {quark }} \neq \mu_{\mathrm{e}}^{\text {hadron }}, P^{\text {quark }}=P^{\text {hadron }}, T^{\text {quark }}=T^{\text {hadron }}$

where $\mu_{\mathrm{B}}^{i}$ and $\mu_{\mathrm{e}}^{i}$ are baryon-number and charge chemical potentials, respectively.

Glendenning [1] claimed, by the bulk calculation, that MC is not appropriate in quarkhadron deconfinement phase transition because of more than one chemical potential, and that there is assumed local charge neutrality instead of total charge neutrality; both hadron matter and quark matter should be charged. Consequently there appears no constant-pressure region like in MC and the structured mixed-phase develops in a wide density region.

The bulk calculation assumes bulk quark matter and hadron matter without any finitesize effect like the surface tension and the Coulomb interaction. Therefore matter is no more uniform but takes geometrical structures in the mixed phase. These geometrical structures are called "droplet", "rod", "slab", "tube" and "bubble."

*endo@ruby.scphys.kyoto-u.ac.jp 
On the other hand, Heiselberg et al [2] pointed out that the mixed phase should appear in a narrow density region and it is energetically disfavored for a large value of the surface tension. In the previous work [3] it has been pointed out that we could satisfy the condition of charge chemical equilibrium even in $\mathrm{MC}$ case by properly introducing the Coulomb potential,

$$
\mu_{\mathrm{e}}^{\text {quark }}=\mu_{\mathrm{e}}^{\text {hadron }}\left(\equiv \mu_{\mathrm{e}}\right)
$$

where $\mu_{\mathrm{e}}^{\text {quark }}-V_{\text {Coul }}^{\text {quark }} \neq \mu_{\mathrm{e}}^{\text {hadron }}-V_{\text {Coul }}^{\text {hadron }}$ which corresponds to Eq. (1) in a gauge invariant fashion.

We can see that the Coulomb screening effect plays an important role and that the geometrical structures become mechanically unstable in the hadron-quark deconfinement phase transition. However, there is used the linearized approximation for the Poisson equation to figure out the Coulomb screening effect analytically.

In this study, we numerically solve the Poisson equation without any approximation. As mentioned above, since the Coulomb potential and its Coulomb screening effect should be so important to change the description of the quark-hadron mixed phase, we must carefully treat the Coulomb interaction.

\section{Formalism}

We use the density functional theory (DFT) to discuss the quark-hadron mixed phase 3]. Consider quark droplets with a radius $R$, embedded in nuclear matter. We divide the whole space into equivalent Wigner-Seitz cells with a radius $R_{\mathrm{W}}$, and impose the total charge neutrality and chemical equilibrium in the quark and hadron phases and at the quark-hadron boundary. The quark phase consists of $u, d, s$ quarks and electrons in chemical equilibrium. To take into account the confinement we incorporate the bag model since we poorly understand the physical picture of the hadron-quark interface. We use the bag constant $B=120 \mathrm{MeV} / \mathrm{fm}^{3}$, which is equal to that in Ref. [2]. As for the hadron phase it consists of nucleons and electrons in chemical equilibrium. We use an effective potential parametrized to reproduce the saturation property of nuclear matter.

At the interface of the quark and hadron phases, we introduce a sharp boundary on the basis of the bag model with the surface tension $\sigma$. Its value should depend on the bag constant and other values such as $s$ quark mass, while we treat it as a free parameter in this study.

Differentiating the thermodynamic potential $\Omega$ with respect to each constituent density $\rho_{i}(i=u, d, s, n, p, e)$ or the Coulomb potential, $V_{\text {Coul }}$, we obtain the equations of motion (EOM) for $\rho_{i}$ and for $V_{\text {Coul }}$. We numerically solve these EOM with the conditions of chemical equilibrium in each phase and at hadron-quark boundary; especially, we fully solve the Poisson equation without any approximation. Note that the Poisson equation becomes highly non-linear because particle charge densities are complicated functions of the Coulomb potential. 

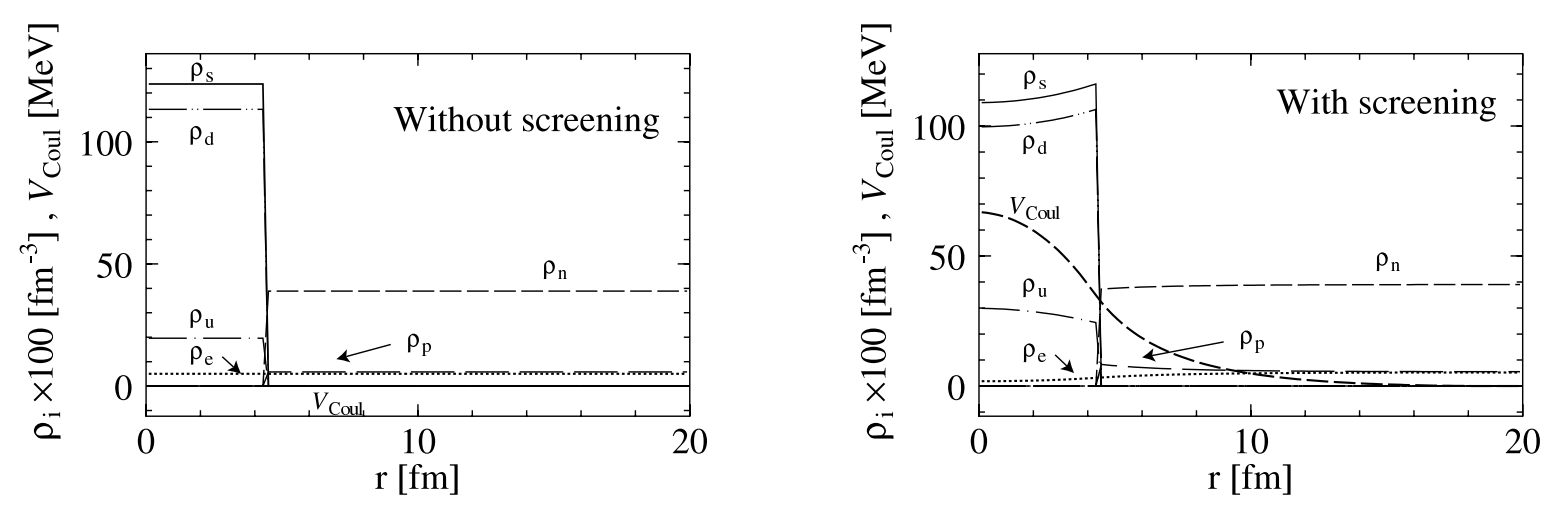

Figure 1. Density profiles $\rho_{i}$ without the Coulomb screening effect (left panel) and with the Coulomb screening effect (right panel). This is the case of $\mu_{\mathrm{B}}=1232 \mathrm{MeV}$ and the volume fraction $f=\left(R / R_{\mathrm{W}}\right)^{3}=0.01$. Each density uniformly spreads in each phase and $V_{\text {Coul }}$ is constant $(=0)$ in the left panel, while there is re-arrangement of the charge densities and $V_{\text {Coul }}$ is spatially dependent in the right panel.

\section{Numerical results}

Figure 1 shows one example of the density profiles without and with the Coulomb screening effect. In the case without the Coulomb screening, each density is uniform in each phase (left panel), while it is not uniform when the Coulomb screening effect is taken into account (right panel). Most remarkable difference between these two figures lies near the hadron-quark interface and the center of the droplet. Negatively charged particle densities are suppressed as they are apart from the boundary; $d, s$ and $e$ in the quark phase and $e$ in the hadron phase. On the contrary, positively charged ones are enhanced; $u$ in the quark phase and $p$ in the hadron phase. As a result, each phase tends to be charge neutral by itself, which should resemble the picture given by MC.

As has been suggested in Ref. [3], the Coulomb screening effect may induce the mechanical instability. In Fig. 2 we demonstrate an example of the case with strong surface tension in our framework. We can see that the Coulomb screening effect strongly suppresses the Coulomb energy for large $R$ and thereby minimum of the thermodynamic potential disappears, which means droplet is mechanically unstable.

We show the phase diagram for the "droplet" phase in Fig. 3, where the droplet can exist as an energetically favored and mechanically stable state. We also depict two curves denoted by "Maxwell" and "Bulk Gibbs" for comparison, which imply the one given by $\mathrm{MC}$ and the one constructed by GC under the ansatz of bulk quark matter and hadron matter, respectively. We can see the $\mu_{\mathrm{B}}-\mu_{\mathrm{e}}$ curve for the droplet phase appears far from "Bulk Gibbs". This feature resembles the case of kaon condensation [4. While the droplet phase is just one of the structured mixed-phases, it could be said that even when there appear other geometrical structures, its $\mu_{\mathrm{B}}-\mu_{\mathrm{e}}$ curve exhibits the similar behavior "Maxwell". Hence, the Maxwell construction may revive the physical meaning effectively. 


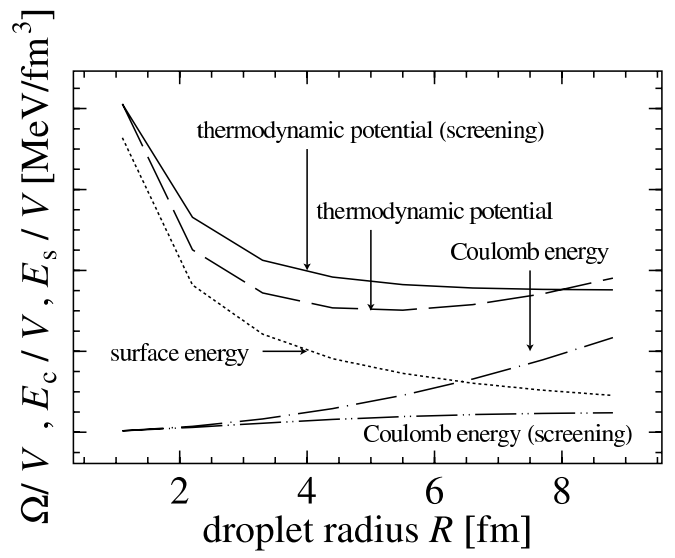

Figure 2. The thermodynamic potential and energy contributions as functions of the droplet radius $R$. This is the case of $\mu_{\mathrm{B}}=1232 \mathrm{MeV}, f=0.01$ and $\sigma=$ $60 \mathrm{MeV} / \mathrm{fm}^{2}$. We can see the Coulomb energy is suppressed by the Coulomb screening effect for large $R$ and the minimum of the thermodynamic potential disappears, which shows the mechanical instability.

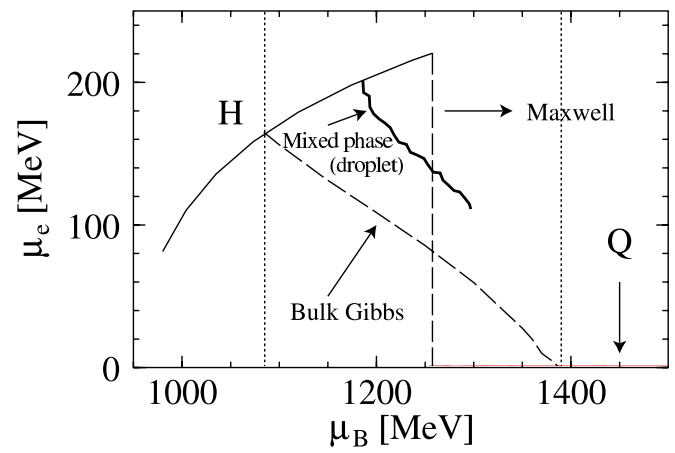

Figure 3. Phase diagram in the $\mu_{\mathrm{B}}-\mu_{\mathrm{e}}$ plane with $\sigma=40 \mathrm{MeV} / \mathrm{fm}^{2}$. In this case, the $\mu_{\mathrm{B}}-\mu_{\mathrm{e}}$ curve for the droplet phase can appear far from "Bulk Gibbs". Note that the $\mu_{\mathrm{B}}-\mu_{\mathrm{e}}$ curve for uniform quark matter denoted by "Q" is lying fairly close to horizontal axis because $\mu_{e} \sim 0$ in uniform quark matter. The $\mu_{\mathrm{B}}-\mu_{\mathrm{e}}$ curve for uniform hadron matter is denoted by " $\mathrm{H}$ ".

\section{Summary}

We have seen the Coulomb screening effect and the finite-size effect in the hadronquark deconfinement phase transition. We have considered here the droplet phase as one of the structured mixed-phases, and found that droplet is mechanically unstable when the surface tension is strong enough. We have found that the Coulomb screening effect as well as the finite-size effect work against the structured mixed-phase and thereby restrict the mixed phase in a narrow density region. It could be suggested, from our work, that there cannot exist wide mixed phase region but only narrow region in neutron star core. However, we have to study other geometrical structures of "rod", "slab", "tube" and "bubble" [5] than "droplet". After this task we can complete the phase diagram in the $\mu_{\mathrm{B}}-\mu_{\mathrm{e}}$ plane.

\section{REFERENCES}

1. N.K. Glendenning, Phys. Rev. D46 (1992) 1274; Phys. Rep. 342 (2001) 393.

2. H. Heiselberg, C. J. Pethick and E. F. Staubo, Phys.Rev.Lett. 70 (1993) 1355

3. D.N. Voskresenky, M. Yasuhira and T. Tatsumi, Phys. Lett. B541 (2002) 93, ; Nucl. Phys. A723 (2003) 291; T. Tatsumi, M. Yasuhira and D.N. Voskresenky, Nucl. Phys. A718 (2003) 359c; T. Tatsumi and D.N. Voskresensky, nucl-th/0312114.

4. T. Maruyama, T. Tatsumi, D.N.Voskresensky, T. Tanigawa and S. Chiba, in this proceedings.

5. T. Endo, T. Maruyama, S. Chiba, T. Tanigawa and T. Tatsumi, in preparation. 